\title{
LOS FACTORES QUE PROPICIAN LA INEJECUCIÓN DE SENTENCIAS EN LOS PROCESOS CONTENCIOSOS ADMINISTRATIVOS SOBRE PAGO DE OBLIGACIONES DINERARIAS EN LOS JUZGADOS MIXTOS DE LA CORTE SUPERIOR DE JUSTICIA DE PUNO EN EL ANO 2012
}

\section{STEPHANIE LIZ CERVANTES ALAGÓN*}

\section{RESUMEN}

Con el desarrollo de la presente investigación, se tiene por objetivo, el análisis de los factores que propician la inejecución de sentencias contencioso administrativas sobre pago de obligaciones dinerarias en los juzgados mixtos de la Corte Superior de Justicia de Puno en el año 2012, el tipo de investigación corresponde al tipo descriptivocuantitativo, puesto que se busca describir los hechos en un solo tiempo conforme a los expedientes analizados, de los cuales se refleja la demasiada carga procesal respecto a procesos contenciosos administrativos que contengan la obligación de dar suma de dinero, los mismos que no tienen un cumplimiento efectivo de la sentencia y no se ven reflejados en la realidad y conforme a ello, proponer alternativas de solución que agilicen la ejecución de sentencias en materia contencioso administrativa.

PALABRAS CLAVE: Administración pública, inejecución de sentencias, obligación de dar, presupuesto anual, proceso contencioso administrativo.

\section{ABSTRACT \\ With the development of this present investigation, it aims to, the analysis of the factors leading to non- execution of administrative litigation judgments on payments of financial obligations in the mixed courts of the Superior Court of Puno in 2012, the type of}

investigation corresponds to the descriptive quantitative, since it seeks to describe the events in a period of time according to the records analyzed, which reflected the excessive caseload regarding the administrative proceedings in which it contain the obligation to give sum of money, They who have no effective enforcement of the judgment and are not reflected in reality and accordingly, to propose alternative solutions to expedite the enforcement of judgments in administrative litigation.

KEYWORDS: Public administration, non-execution of judgments, obligation to give, annual budget, administrative proceedings

\section{INTRODUCCIÓN}

El Proceso Contencioso Administrativo, se encuentra regulado por el T.U.O. de la Ley General de Proceso Contencioso Administrativo D.S. No 013-2008-JUS; y conforme a las pretensiones contenidas en el artículo $5^{\circ}$ de la Ley NN$^{0} 27444$ «Ley del Procedimiento Administrativo General», es susceptible de impugnarse todo acto que es realizado en el ejercicio de las potestades administrativas.

Los procesos contenciosos administrativos tramitados en los Juzgados Mixtos de Puno, en su mayoría obedecen al pago de obligaciones dinerarias

* Bachiller de la Facultad de Ciencias Jurídicas y Políticas, Escuela Profesional de Derecho de la UNA- PUNO. 
por parte del Estado, quien es el principal deudor, y quien no ejecuta las sentencias contenciosas administrativas; con la investigación realizada se pretende dar respuesta al siguiente problema, ¿cuáles son los factores que propician la inejecución de sentencias contencioso administrativas sobre pago de obligaciones dinerarias en los Juzgados Mixtos de la Corte Superior de Justicia de Puno?, de ésta forma, se identifica a las instituciones públicas que inciden en la inejecución de sentencias contenciosas administrativas y se determina cuál es la normatividad aplicable en la ejecución de sentencias contenciosas administrativas.

Primero se aborda: el planteamiento teórico, la determinación del problema, su justificación, la determinación de los objetivos, hipótesis y las variables, que son la estructura de ésta investigación. En segundo lugar, se trata: el diseño metodológico utilizado de la investigación, el tipo, el ámbito de estudio, el universo y la muestra. Finalmente, se presentan los resultados, teniéndose como factores que propician la inejecución de sentencias, las actuaciones dilatorias de la entidad demandada, la falta de presupuesto y la deficiencia normativa, encontrándose que el $92 \%$ de sentencias no se ejecutan en el año 2012, así como, la entidad más deudora en Puno es la DREP, para ello se propone como alternativa de solución determinar un plazo razonable para la ejecución de sentencias, así como realizar la indemnización cuando se supere el plazo, de tal manera, no vulnerar el derecho a la tutela jurisdiccional efectiva contenido en el artículo 139 inciso 2 de la Constitución Política del Perú y generar confianza en la Administración Pública. Conforme a lo vertido en los párrafos precedentes acerca del contenido de la presente investigación, se da inicio a su desarrollo esperando que cumpla con las expectativas del lector.

\subsection{OBJETIVOS}

\subsubsection{Objetivo general}

Establecer qué factores propician el incumplimiento 0 inejecución de sentencias sobre pago de obligaciones dinerarias.

\subsubsection{Objetivos específicos}

Identificar que instituciones públicas inciden en la inejecución de sentencias contencioso administrativas.

Determinar la normatividad aplicable en la ejecución de sentencias contenciosas administrativas sobre pago de obligaciones dinerarias.

\subsection{HIPÓTESIS}

\subsubsection{Hipótesis general}

Conforme a la cantidad de procesos contenciosos administrativos tramitados en los Juzgados Mixtos de Puno. Es probable que los factores que propician la inejecución de las sentencias lo constituya la deficiencia normativa, la falta de presupuesto y las actuaciones dilatorias de la entidad del Estado demandada.

\subsubsection{Hipótesis específica}

La administración pública influye en la inejecución de sentencias, siendo las que predominan: DREP y ONP.

El proceso contencioso administrativo se regula mediante la Ley $\mathrm{N}^{\circ} 27584$, modificado por el Decreto Supremo N 013-2008-JUS.

\section{MATERIALES Y MÉTODOS}

\subsection{Diseño de investigación}

La investigación corresponde a una metodología del tipo cuantitativo, porque se efectuará el análisis de los factores que propician la inejecución de sentencias en los procesos contenciosos administrativos en los juzgados mixtos de la Corte Superior de Justicia de Puno durante el año 2012. El enfoque cuantitativo pretende obtener la recolección de datos para conocer o medir el fenómeno en estudio y encontrar soluciones para la misma, la cual trae consigo la afirmación o negación de la hipótesis establecida en dicho estudio (Hernández Sampieri, Fernández Collado y Baptista Lucio, 2006, pág. 8). 
Siendo una investigación jurídica, corresponde al tipo jurídico-social, porque está encaminada a dar una solución sobre las obligaciones dinerarias del Estado hacia el administrado, de la misma forma corresponde a un diseño metodológico no experimental, toda vez que la relación de los datos no es objeto de manipulación deliberada, es decir, observamos los hechos tal como se dan, y se procede a realizar un análisis de dichos hechos en un solo momento 0 en un tiempo único, por lo que es transeccional o transversal, el estudio es del tipo descriptivo, recolectando información sobre cada una de las variables del problema existente sobre la ejecución de sentencias en los procesos contenciosos administrativos.

\section{2. Ámbito de estudio}

El ámbito de estudio se concentra en las sentencias emitidas por los juzgados mixtos constituidos por el primer, segundo y tercer juzgado mixto de la Corte Superior de Justicia de Puno.

\subsection{Universo y muestra}

\subsubsection{Universo}

Las unidades de análisis de la investigación son los expedientes que se tramitaron, ejecutaron y archivaron en el Distrito de Puno, en el periodo del 2012

\subsubsection{Muestra}

Se tomaron indistintamente 100 expedientes de procesos contenciosos administrativos ejecutados y archivados del año judicial 2012.

Además, se utilizaron aportes textuales para la elaboración de la presente investigación, tales como: libros realizados por diferentes juristas dentro de nuestro medio nacional, el Informe Defensorial $\mathrm{N}^{\circ}$ 19 y 121 realizados por la Defensoría del Pueblo, publicaciones en internet en cuanto a obligaciones por parte del Estado en materia Contencioso Administrativo.

\section{DISCUSION TEÓRICA}

\subsection{Antecedentes}

Los antecedentes investigativos más relevantes acerca de la ejecución de sentencias contenciosas administrativas recaen en el artículo de Miguel Rojas Bernal (Rojas Bernal, 2013) en la publicación de Gaceta Constitucional sobre "Cobrarle al Estado: régimen de ejecución de sentencias constitucionales y posibilidades de actuación judicial». En este artículo, el autor se avoca de manera específica a las posibilidades que tienen los jueces constitucionales para actuar este tipo de sentencias condenatorias. Considera que en el marco jurídico, las normas del Código Procesal Constitucional, los principios de: dirección judicial del proceso, impulso de oficio, aformalismo y socialización, también aplicables en etapa de ejecución, corresponde a los jueces constitucionales encontrar las soluciones que les permitan sortear los obstáculos para lograr el cumplimiento de sus mandatos.

De la misma manera, se encontró el informe № 19 de la Defensoría del Pueblo (Defensoria del Pueblo, 2013), al respecto del Incumplimiento de Sentencias por Parte de la Administración Estatal, en cuanto a las pretensiones de contenido patrimonial y los mandatos judiciales distintos a la reposición. De éstos, las acreencias laborales ocupan el primer lugar, seguidas de las obligaciones de pago o nivelación de pensiones. Frente a estos requerimientos, los entes públicos han respondido de diversa manera. En algunos casos se procede al cumplimiento total 0 parcial, en otros se opone una simple negativa, llegando incluso a no informar adecuadamente sobre las razones del incumplimiento. También se aduce la necesidad del cumplimiento previo de un requisito administrativo, así como la imposibilidad jurídica 0 fáctica de cumplir conforme al Informe Defensorial № 121 «Propuestas para una reforma de la Justicia Contencioso-Administrativa desde la perspectiva del acceso a la justicia» http://www.defensoria.gob.pe/ informes-publicaciones.php (Defensoria del Pueblo, 2013)

Recientemente, resulta de importancia la publicación realizada el 24 de junio del 2014 en el diario «El Peruano», suplemento Jurídica realizado por el Maestro José Antonio Tirado, titulado «En el Ámbito 
del Proceso Contencioso Administrativo-Ejecución de Sentencias», en la cual adopta un criterio constructivo acerca fijar la forma de ejecución de una sentencia condenatoria, la cual debería estar organizada a partir de tres pilares: prevenir las dificultades de ejecución de las sentencias, facilitar e impulsar su cumplimiento y reprimir la infracción cometida por los responsables de la inejecución. (Tirado, 2014, pág. 2).

\subsection{Discusión de Resultados}

El procedimiento que se realiza para la ejecución de obligaciones de dar suma de dinero, se encuentra regulado dentro del artículo 47 del D.S. N ${ }^{0}$ 013-2008JUS, que dicho sea de paso, ha sufrido la modificación del inciso 3 por la Ley $N^{0} 30137$, ley que establece criterios de priorización para la atención del pago de sentencias judiciales. Por lo que, para ser efectivo, se requiere que la sentencia adquiera la calidad de cosa juzgada, y la oficina general de Administración (entiéndase como la entidad demandada) procederá conforme al mandato judicial dentro de las "Leyes de Presupuesto». (Monzon Valencia de Echevarría, 2011, pág. 245), al respecto indica que, "Eso significa que sólo puede pagar, con el dinero disponible en el rubro sentencias judiciales consentidas; por tanto no se puede cumplir lo ordenado en la sentencia con fondos establecidos para otros rubros», lo que acarrearía de hacerlo en responsabilidad funcional por parte de quien destina de otros fondos. Sin embargo, cuando resultase insuficiente el financiamiento, previa evaluación y priorización, el titular del pliego presupuestario hace de conocimiento su compromiso de atender las sentencias de conformidad con el artículo 70 de la Ley $\mathrm{N}^{0} 28411$.

Véase que, la ley no dice que pagará, sino que previa evaluación y priorización el titular podrá realizar modificaciones presupuestarias. Con lo cual se infiere que, si hay presupuesto disponible pero hay otras prioridades no prosperará el pago en ese periodo; mas, si no perjudica las demás partidas o rubros de la entidad entonces sí podría cumplirse con lo ordenado por el Juez. (Monzon Valencia de Echevarría, 2011, pág. 426)

Ahora bien, el artículo 70 de la Ley 28411, expresa que: «70.1 Para el pago de sumas de dinero por efecto de sentencias judiciales en calidad de cosa juzgada, se afecta hasta el cinco por ciento (5\%) 0 hasta un mínimo de tres por ciento (3\%), según sea necesario, de los montos aprobados en el Presupuesto Institucional de Apertura (PIA), con excepción de los fondos públicos correspondientes a las fuentes de financiamiento Donaciones y Transferencias y Operaciones Oficiales de Crédito Interno y Externo, la reserva de contingencia y los gastos vinculados al pago de remuneraciones, pensiones y servicio de tesorería y de deuda. Esta norma comprende, entre otros, la atención de sentencias judiciales en calidad de cosa juzgada por adeudos de beneficios sociales.

70.2 El Ministerio de Economía y Finanzas, a través de la Dirección Nacional del Tesoro Público, procederá a la apertura de una cuenta bancaria en el Banco de la Nación para cada Entidad que lo solicite, en la cual la Entidad deberá depositar, mensualmente, los montos de las afectaciones presupuestales mencionadas en el numeral precedente, bajo responsabilidad del Director General de Administración o quien haga sus veces en la Entidad.

70.3 Los pagos de las sentencias judiciales, incluidas las sentencias supranacionales, deberán ser atendidos por cada Entidad, con cargo a su respectiva cuenta bancaria indicada en el numeral precedente, debiendo tomarse en cuenta las prelaciones legales.

70.4 En caso de que los montos de los requerimientos de obligaciones de pago superen el porcentaje señalado en el numeral 70.1 del presente artículo, la Entidad debe cumplir con efectuar el pago en forma proporcional a todos los requerimientos existentes de acuerdo a un estricto orden de notificación, hasta el límite porcentual.

70.5 Los requerimientos de pago que superen los fondos públicos señalados en el numeral $70.1 \mathrm{del}$ presente artículo se atenderán con cargo a los presupuestos aprobados dentro de los cinco (5) años fiscales subsiguientes.

70.6 Precísese que la prelación legal, implica que las deudas se cancelan y/o amortizan bajo responsabilidad, priorizando la antigüedad del expediente que contiene el monto adeudado, así 
como la presentación de la documentación sustentatoria».

Al respecto el artículo $70^{\circ}$, si bien es cierto dispone la forma de pago para obligaciones dinerarias, en la cual, las entidades deben de aperturar una cuenta bancaria, y si los requerimientos superasen los fondos públicos se atenderán dentro de los 5 años fiscales siguientes, sin embargo, tener que esperar hasta cinco años resulta una larga espera de la que pende la ejecución efectiva de la sentencia, sobre ello, la Defensoría del Pueblo en su Informe Defensorial $\mathrm{N}^{0} 121$, indica que el promedio de vida de una persona según estudios realizados por el INEI es de 70 años, y teniéndose en cuenta que un pensionista alcance fundada su demanda a los 67 el plazo para su ejecución sería cuando ya éste haya fallecido.

De los expedientes analizados tenemos que en su mayoría no son ejecutados, pese a tener una sentencia fundada en derecho, ésta sólo queda en el limbo de las decisiones justas, incluso el tiempo transcurrido en exceso del plazo casi esbozado por la ley de cinco años, resulta ser extenso y no se compensa. Por ello el plazo para la ejecución debe establecerse en forma determinada sin distinción, de tal manera que las Entidades que son del Estado cumplan, y sin en el caso que transgredieran el plazo nuevo señalado, se deberá proceder al pago de una indemnización compensando el tiempo en exceso perdido, además de sancionarse al titular del pliego responsable.

Ésta inejecución de sentencias contenciosas administrativas afecta principalmente al derecho de tutela jurisdiccional efectiva, la misma que se encuentra constitucionalmente reconocida en el artículo 139.3 de la Constitución Política del Perú, entiéndase a la tutela jurisdiccional efectiva como indica (Huapaya Tapia, 2006, pág. 392) «es un derecho humano de acceso a la justicia, un derecho a que cada persona tenga derecho a un recurso efectivo para la protección de sus derechos e intereses, y a que ésta protección sea judicial, plena y efectiva».

En ese orden de ideas, Monroy Gálvez citado por (Ticona Postigo, 1998), señala que, "es uno de carácter público y subjetivo por el que toda persona, por el solo hecho de serlo, está facultada para exigir al Estado tutela jurídica plena; se manifiesta de dos maneras: el derecho de acción y de contradicción».

(Priori Posada, 2009, pág. 771), Priori Posada acertadamente indica, que, "es el derecho constitucional que tiene todo sujeto de derecho de acceder a un órgano jurisdiccional para solicitar tutela de una situación jurídica que se alega está siendo vulnerada o amenazada a través de un proceso dotado de las mínimas garantías, luego del cual se expedirá una resolución fundada en derecho, que desplegará efectos vinculantes».

El hecho de no verse ejecutada la sentencia, o no ser ejecutada dentro de un plazo razonable, o que esta no se ejecute, afecta además al derecho a la efectividad de la sentencia, ya que, la sentencia se considerará efectiva en la medida que ha sido obtenida como resultado de un debido proceso, y el mandato que contiene, es cumplido en forma íntegra por las partes. Como señala (Priori Posada, 2009, pág. 80) «no se trata pues de que el juez expida la sentencia y que la decisión contenida en ella quede en el papel. Por el contrario, la sentencia será efectiva en tanto que las partes cumplan en la realidad, en la práctica con lo ordenado por el Juez».

El Tribunal Constitucional en el expediente N031492004-AC/TC, cita el Caso Hornby c/ Grevia del Tribunal Europeo de Derechos Humanos, donde expresa que la efectividad de la sentencia forma parte de las garantías judiciales, pues sería ilusorio que una decisión judicial quedase inoperante causando daño a las partes.

Ergo, si el derecho a la ejecución de las decisiones judiciales, como bien se ha expresado en el fundamento 16 de la sentencia contenida del expediente STC N4080-2004-AC/TC, al referir que, «El derecho a la ejecución de las decisiones jurisdiccionales resulta de especial relevancia no sólo por su manifestación de derecho de tutela judicial, sino porque constituye una garantía sine qua non para que pueda evidenciarse, en la práctica, el principio de independencia judicial, que conforme lo ha declarado este Tribunal no es sólo uno de los elementos (...) que, conforme al artículo 43 de la Constitución, nos configuran como una República Democrática, sino que, además, resulta (...) necesaria 
(o) para inspirarla confianza de los ciudadanos en los tribunales» (Expediente $\mathrm{N}^{\circ}$ 0004-2004-CC/ TC, ff., 33). Si las sentencias de los jueces no se cumplen, simplemente no podría hablarse de un Poder Judicial independiente que es capaz de hacer valer su jurisdictio con plena eficacia respecto de lo que decide, y de este modo, los ciudadanos no tendrían un garante real para la protección de sus derechos.

Muchas de las entidades del Estado, no ejecutan precisamente arguyendo que no tienen el presupuesto suficiente, o realizando escritos con el afán de prorrogar más la ejecución de la sentencia, invocando incluso el principio de legalidad presupuestaria contenido en el artículo 77 de la Constitución, sin embargo, éste principio no puede ser usado para justificar el incumplimiento de las sentencias judiciales, en ese sentido el TC en el Expediente $\mathrm{N}^{0}$ 3149-2004-AC/TC en su fundamento 8 señala que, "Carece de toda racionalidad, si se tiene en cuenta que el propio Estado, a través de su presupuesto público es quien solventa los gastos de los procuradores públicos y abogados que acuden a defender a los funcionarios emplazados con la demanda argumentando que no existe presupuesto o teniendo la voluntad de cumplir deben de esperar a la programación del MEF. Siendo una práctica sistemática y reiterada de agresión al personal docente».

Valga decir, que en la ciudad de Puno, los procesos que en su mayoría son demandantes los docentes, que son afectados con la inejecución de sus sentencias.

\subsection{Resultados}

Los siguientes cuadros y gráficos, que son empleados en esta investigación, contienen la información recolectada de los expedientes: del primer, segundo y tercer juzgado mixto, mediantes los cuales permitiremos mostrar los resultados y realizar la comprobación de las hipótesis planteadas.

Por medio gráfico número uno (1), se presentan la cantidad de expedientes que han sido materia de análisis y estudio, con predominación del 2 do Juzgado Mixto de la Corte Superior de Justicia de Puno, el cual tiene mayor cantidad de procesos contenciosos administrativos que se encuentran pendientes de ejecución de sentencia, seguido por el primer juzgado mixto y finalmente por el tercer juzgado mixto de la Corte Superior de Justicia de Puno.

\section{GRÁFICO Nº 01}

\section{TÍTULO: RELACIÓN DE EXPEDIENTES CONTENCIOSOSADMINISTRATIVOS ANALIZADOS.}

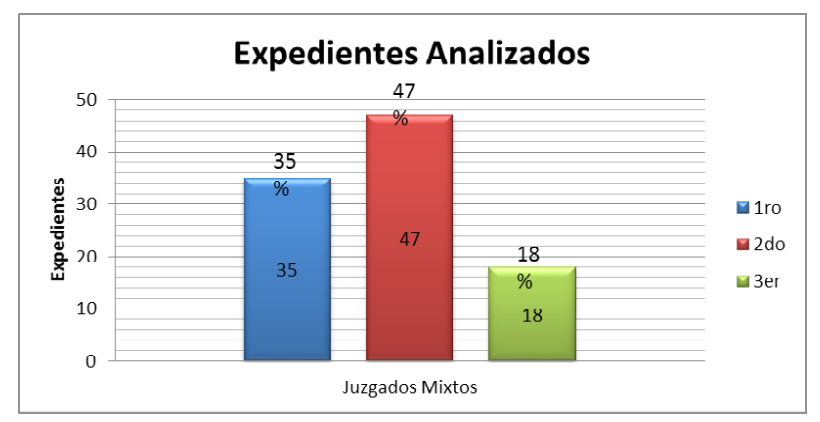

${ }^{*}$ Fuente: Fichas de Observación

\subsubsection{En cuanto a las variables independientes}

Las variables independientes de la presente investigación son: la deficiencia normativa, falta de asignación de presupuesto y las actuaciones dilatorias de la entidad demandada. Por medio de ésta sección presentaré los cuadros y gráficos, los cuales desarrollan los resultados obtenidos mediante la presente investigación.

\section{1. variable independiente-deficiencia normativa}

En cuanto a la variable deficiencia normativa, para poder realizar su verificación tenemos los siguientes indicadores: las medidas coercitivas empleadas y el plazo de ejecución.

Mediante el cuadro $\mathrm{N}^{0} 02$ se aprecian los indicadores de las medidas coercitivas empleadas. De la cual se evidencia de todos los expedientes analizados, únicamente ocho remite copias al Ministerio Público para que pueda ser efectuadas y a la par incluyen la imposición de multas acumulativas por no darse el cumplimiento de la sentencia en un plazo razonable, sin embargo, se tiene una alarmante cifra en cuanto noventa y dos expedientes en las que no se habría aplicado una medida coercitiva que permita dar un cumplimiento de la sentencia. 
CUADRO $\mathrm{N}^{\circ} 02$

TÍTULO: MEDIDAS COERCITIVAS EMPLEADAS EN LOS PROCESOS CONTENCIOSOS ADMINISTRATIVOS

\begin{tabular}{|c|r|r|}
\hline MEDIDAS COERCITIVAS & CANTIDAD DE EXP. & \multicolumn{1}{c|}{ PORCENTAJE } \\
\hline Remisión de copias al Ministerio Público & 8 & $8 \%$ \\
\hline Imposición de multas acumulativas & 8 & $8 \%$ \\
\hline Ninguna medida & 92 & $92.0 \%$ \\
\hline
\end{tabular}

${ }^{\star}$ Fuente: Expedientes del 1er, 2do y 3er Juzgados Mixtos de la Corte Superior de Justicia de Puno en el año 2012.

\section{CUADRO N ${ }^{\circ} 03$}

\section{TÍTULO: PLAZO DE EJECUCIÓN}

\begin{tabular}{|c|c|c|}
\hline PLAZO DE EJECUCIÓN & CANTIDAD DE EXP. & PORCENTAJE \\
\hline De 1 mes a 8 meses & 1 & $1 \%$ \\
\hline De 9 meses a 12 meses & 4 & $4 \%$ \\
\hline De 1 año a más & 3 & $3.0 \%$ \\
\hline No se precisa & 92 & $92.0 \%$ \\
\hline \multicolumn{2}{|l|}{ Total } & $100.0 \%$ \\
\hline
\end{tabular}

${ }^{\star}$ Fuente: Expedientes del 1er, 2do y 3er Juzgados Mixtos de la Corte Superior de Justicia de Puno en el año 2012.

Mediante el cuadro $\mathrm{N}^{0} 03$ se detalla el plazo de ejecución de la sentencia, como se observa el ocho por ciento precisa un plazo para su cumplimiento, sin embargo, en su mayoría no hay un plazo preciso para el cumplimiento de la sentencia, por lo que el proceso finalmente se archiva.

\section{2. variable independiente- falta de asignación de presupuesto}

La segunda variable independiente se refiere a la falta de asignación de presupuesto, variable que será verificada mediante el indicador de, los oficios de requerimiento de presupuesto que se han realizado, de tal manera nos permita conocer si la parte demandada ha comunicado que no puede cumplir la sentencia o dilatar el proceso aduciendo la falta de presupuesto.

\section{CUADRO N 04}

TÍTULO: OFICIOS DE FALTA DE PRESUPUESTO

\begin{tabular}{|c|r|r|}
\hline ESCRITOS & CANTIDAD DE EXP. & PORCENTAJE \\
\hline Falta de Presupuesto Anual & 83 & $83 \%$ \\
\hline presupuesto para el sgte año & 17 & $17 \%$ \\
\hline Total & & $100 \%$ \\
\hline
\end{tabular}

Fuente: Expedientes del 1er, 2do y 3er Juzgados Mixtos de la Corte Superior de Justicia de Puno en el año 2012.

Se ha observado además que las entidades del Estado, se han mostrado renuentes a dar cumplimiento a la sentencia que dispone fundada la demanda de parte, alegando que no tienen el presupuesto suficiente para cubrir con el pago de la sentencia, argumento que no debe ser considerado como excusa para no dar su cumplimiento.

\section{3. variable independiente- actuaciones dilatorias de la entidad demandada}

Para comprobar los resultados en esta sección sobre nuestra tercera variable independiente denominada "actuaciones dilatorias de la entidad demandada», el indicador que permite visualizar los resultados es, los escritos presentados ante el juzgado. Lo cual se describe en el cuadro y gráfico $\mathrm{N}^{0} 05$.

\section{CUADRO N०05}

TÍTULO: ACTUACIONES DILATORIAS DE LA ENTIDAD DEMANDADA

\begin{tabular}{|c|r|r|}
\hline ESCRITOS & CANTIDAD DE EXP. & PORCENTAJE \\
\hline Falta de Presupuesto & 83 & $83 \%$ \\
\hline Proposición de excepciones & 13 & $13 \%$ \\
\hline Otros & 4 & $4 \%$ \\
\hline
\end{tabular}

Fuente: Expedientes del 1er, 2do y 3er Juzgados Mixtos de la Corte Superior de Justicia de Puno en el año 2012.

Las actuaciones dilatorias son aquellos actos que realiza la entidad del Estado que es demanda, con la finalidad de no cumplir el mandato de la sentencia, conforme se ha podido recolectar las entidades realizan: escritos repetitivos indicando la falta de presupuesto, la proposición de excepciones, y la denuncia civil, todo ello conforme a dilatar más la actuación oportuna y razonable de un proceso.

\subsubsection{En cuanto a las variables dependientes}

Las variables dependientes de la presente investigación son: inejecución de sentencias contenciosas sobre pago de obligaciones dinerarias de las entidades de la administración pública y nivel de ejecución de sentencias contencioso administrativas. Mediante los cuadros y gráficos, se mostraran los resultados obtenidos mediante la ejecución de la investigación.

1. variable dependiente- inejecución de sentencias contenciosas sobre pago de obligaciones dinerarias de las entidades de la administración pública

La comprobación de la primera variable dependiente es la inejecución de las sentencias contenciosas administrativas sobre pago de obligaciones dinerarias de las entidades de la administración pública. 
Mediante el cuadro $\mathrm{N}^{0} 06$, se detallará cuántos expedientes se han ejecutado y cuántos de los expedientes contenciosos administrativos no se han ejecutado en el año 2012, para lo cual el indicador, lo constituyen los expedientes que han sido tramitados y archivados durante el año judicial 2012 en la Corte Superior de Justicia de Puno.

\section{CUADRO N ${ }^{\circ} 06$}

\section{TÍTULO: INEJECUCIÓN DE SENTENCIAS CONTENCIOSAS ADMINISTRATIVAS}

\begin{tabular}{|c|r|r|}
\hline EJECUCIÓN DE PAGO & CANTIDAD DE EXP. & PORCENTAJE \\
\hline Ejecución de la sentencia & 8 & $8 \%$ \\
\hline Inejecución de la sentencia & 92 & $92 \%$ \\
\hline Total & & $100 \%$ \\
\hline
\end{tabular}

Fuente: Expedientes del 1er, 2do y 3er Juzgados Mixtos de la Corte Superior de Justicia de Puno en el año 2012.

Conforme al cuadro apreciado, se tiene que la mayoría de procesos no se ejecutan, y los pocos que se han ejecutado son porque ha existido persistencia de parte de los demandantes, así como la imposición de un plazo en la ejecución de la sentencia, además de la cantidad de apercibimientos efectivos.

\section{2. variable dependiente- nivel de ejecución de sentencias contenciosas administrativas}

La comprobación de la primera variable dependiente, correspondiente al nivel de ejecución de sentencias contenciosas administrativas, los indicadores se encuentran constituidos, por las entidades de la administración pública que han sido demandados de forma renuente, conforme se tiene esbozado en el cuadro $\mathrm{N}^{0} 07$ referido a las entidades deudoras.

\section{CUADRO N 07 \\ TÍTULO: ENTIDADES DEUDORAS}

\begin{tabular}{|c|r|r|}
\hline ENTIDADES DEUDORAS & NÚMERO & \multicolumn{1}{|c|}{ PORCENTAJE } \\
\hline DREP & 91 & $91 \%$ \\
\hline DRES & 6 & $6 \%$ \\
\hline ONP & 1 & $1 \%$ \\
\hline DRAP & 1 & $1 \%$ \\
\hline BENEFICENCIA PÚBLICA & 1 & $1 \%$ \\
\hline TOTAL & & $100.0 \%$ \\
\hline
\end{tabular}

Fuente: Expedientes del 1er, 2do y 3er Juzgados Mixtos de la Corte Superior de Justicia de Puno en el año 2012.

Del cuadro que antecede, la Entidad del Estado que es más deudora, lo constituye la DREP, quien es renuente al pago de bonificaciones especiales, donde la mayoría de partes afectadas son docentes del sector educación.

\section{CUADRO N ${ }^{\circ} 08$}

TÍTULO: NORMATIVIDAD APLICABLE EN LA EJECUCIÓN DE SENTENCIAS CONTENCIOSAS ADMINISTRATIVAS

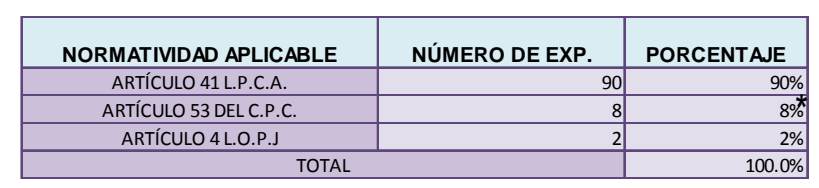

Fuente: Expedientes del 1er, 2do y 3er Juzgados Mixtos de la Corte Superior de Justicia de Puno en el año 2012.

En cuanto a la normatividad aplicada en los procesos contenciosos administrativos, no realizan apercibimientos contenidos en el artículo 53 del Código Procesal Civil, así como el artículo 46 del LPCA e incluso del artículo 4 de la L.O.P.J., mediante la cual se indica que todas las resoluciones emitidas deben de ser cumplidas.

\section{CONCLUSIONES}

Los factores que propician la inejecución de sentencias sobre pago de obligaciones dinerarias en los procesos contenciosos administrativos son: las actuaciones dilatorias de las entidades del Estado demandadas, la falta de presupuesto y la deficiencia normativa, siendo la más recurrente las actuaciones dilatorias de la entidad demandada, quienes realizan este procedimiento para evadir el cumplimiento de la sentencia y alargan el mismo procedimiento.

La entidad administrativa del Estado más deudora, es la Dirección Regional de Educación de Puno. La deuda es respecto de los docentes que vencieron judicialmente sobre el pago por preparación de clases y evaluación correspondiente al $30 \%$ de su remuneración total, cuya inejecución de sentencias, no sólo genera la desconfianza en la Administración Pública, sino además vulnera el derecho a la tutela jurisdiccional efectiva contenido en el artículo 139 inciso 3 de la Constitución Política del Perú.

Con la implementación de la Ley $N^{\circ} 30137$, ley que establece criterios de priorización para la atención para el pago de sentencias judiciales, ha modificado el artículo 47 inciso 3 de la L.G.P.C., estableciéndose que el plazo para el pago de sentencias se debe realizar conforme al artículo $70^{\circ}$ de la Ley 28411 Ley General Nacional de Presupuesto, la cual si bien es cierto, establece criterios para el pago según edad, fecha de notificación, materia y monto obligacional, 
sin embargo, no se implementado una medida que tienda a indemnizar a los beneficiarios de la sentencia por el retardo de la ejecución de la misma.

\section{RECOMENDACIONES}

Con la dación de la Ley 30137, se establecen los criterios de priorización para la atención del pago de sentencias judiciales, modifica el artículo 47 inciso 3 , en el sentido de que cuando se supere las posibilidades de financiamiento, se regirá conforme al artículo $70^{\circ}$ de la Ley 28411 , donde dispone lineamientos sobre el pago de sentencias judiciales, el cual afecta el 3\% hasta el $5 \%$ del Presupuesto Institucional de Apertura, margen que resultan insuficientes para cubrir la ejecución de sentencias, además no contiene un plazo de ejecución fijo, por lo que se recomienda modificar el artículo $70^{\circ}$ el cual debe decir, «70.1. Para el pago de sumas de dinero por efecto de sentencias judiciales en calidad de cosa juzgada, se afectará hasta el siete por ciento (7\%) o hasta un mínimo de tres por ciento (3\%), según sea necesario, de los montos aprobados en el Presupuesto Institucional de Apertura (PIA), con excepción de los fondos públicos...».

Se ha observado que en la mayoría de casos, no se le considera al beneficiado con la sentencia: el daño que ha sido ocasionado con el desgaste del tiempo y la espera en su ejecución. Por tanto, debe de regularse el procedimiento que debería llevarse a cabo para calcular la cuantificación del daño o perjuicio ocasionado por el incumplimiento. Procedimiento que debería incluir el artículo $70^{\circ}$ en un nuevo inciso. «Artículo 70. 7. Transcurrido el plazo de 3 años para ejecutar la sentencia, la Entidad demandada deberá efectuar la indemnización correspondiente, por el incumplimiento del pago oportuno en la ejecución de la sentencia con calidad de cosa juzgada, bajo responsabilidad penal y administrativa del titular del pliego»

\section{BIBLIOGRAFIA}

Defensoria del Pueblo. (2013). Propuesta para una reforma de la Justicia Contenciosa Administrativadesde la perspectiva del Acceso a la Justicia. Lima:Defensoria del Pueblo.
Defensoria del Pueblo (2013). incumplimiento de sentencias por parte de la administración estatal, aprobado por Resolucion Defensorial $N^{\circ}$ 62-98/DP. Lima: Defensoria del Pueblo.

Hernández Sampieri, Fernández Collado y Baptista Lucio. (2006). Metodologia de la Investigación . Mexico.

Huapaya Tapia, R. (2006). Tratado de Proceso Contencioso Administrativo. Lima: Jurista Editores.

Monzon Valencia de Echevarría, L. L. (2011). Comentario Exegético a la Ley que regula el Proceso Contencioso Administrativo. Lima: Ediciones Legales.

Priori Posada, G. (2009). Comentarios a la ley del Proceso Contencioso Administrativo. Lima: Ara Editores.

Rojas Bernal, M. (2013). Cobrarle al estado: régimen de ejecucion de sentencias constitucionales y posibilidades de actuación judicial. Gaceta Constitucional.

http://www.gacetaconstitucional.com.pe/sumario.

Ticona Postigo, V. (1998). Análisis y comentarios al Código Procesal Civil. Lima: San Marcos.

Tirado, J. A. (24 de Junio de 2014). En el Ámbito del proceso Contencioso Administrativo- Ejecución de Sentencias. Jurídica- El Peruano, pág. 2.

\section{JURISPRUDENCIA}

1. EXP. N ${ }^{\circ} 4080-2004-A C / T C$. Consulta: 08-10-13

2. EXP. No 3149-2004-AC/TC. Consulta: 08-10-13 